Supporting Information for :

\title{
One-pot synthesis of superfine core-shell Cu@metal nanowires for highly tenacious transparent LED dimmer
}

Huachun Wang ${ }^{1}$, Chenping Wu ${ }^{1}$, Youyang Huang ${ }^{1}$, Feipeng Sun ${ }^{1}$, Na Lin ${ }^{1,2}$, Abdul

Majid Soomro ${ }^{1}$, Zhibai Zhong ${ }^{1}$, Xiaodong Yang ${ }^{3}$, Xiaohong Chen ${ }^{1, *}$, Junyong Kang ${ }^{1}$, and Duanjun $\mathrm{Cai}^{1,4, *}$

${ }^{1}$ Fujian Key Laboratory of Semiconductor Materials and Applications, CI center for OSED, College of Physical Science and Technology, Xiamen University, Xiamen 361005, China.

${ }^{2}$ Xiamen Industrial \& Commercial School, Jimei district, Xiamen 361024, China.

${ }^{3}$ State Key Laboratory of Physical Chemistry of Solid Surfaces, College of Chemistry and Chemical Engineering, Xiamen University, Xiamen 361005, China.

${ }^{4}$ Department of Chemistry, Duke University, Durham, NC 27708-0354, U.S.A.

* Corresponding Author E-mail: xhchen@xmu.edu.cn, dcai@xmu.edu.cn 


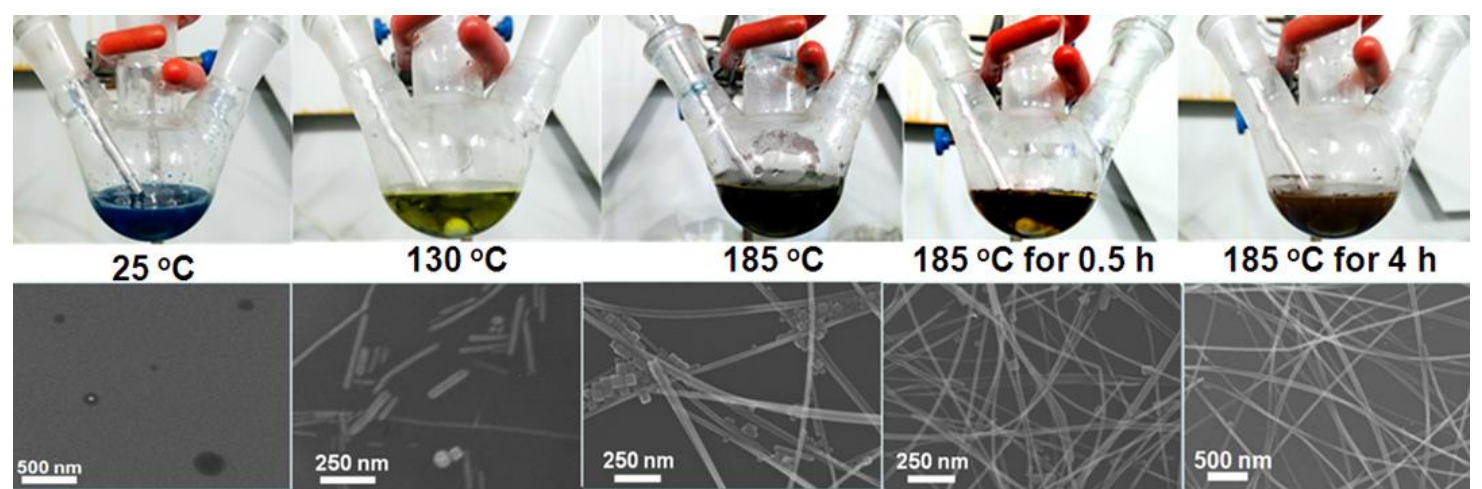

Fig. S1. Stage illustration for the growth of $\mathrm{Cu}$ NWs. From $\sim 130{ }^{\circ} \mathrm{C}$, the short $\mathrm{Cu}$ NWs begin to form with small seeds. At $\sim 185{ }^{\circ} \mathrm{C}$, large amount of $\mathrm{Cu}$ cubic seeds form and long $\mathrm{Cu} \mathrm{NWs}$ reach a length of 40 um with uniform diameter. As reaction time goes on, those cubic seeds are dispersed by the ostwald ripening process and complete yield of unifom $\mathrm{Cu}$ NWs could be obtained after $4 \mathrm{~h}$.
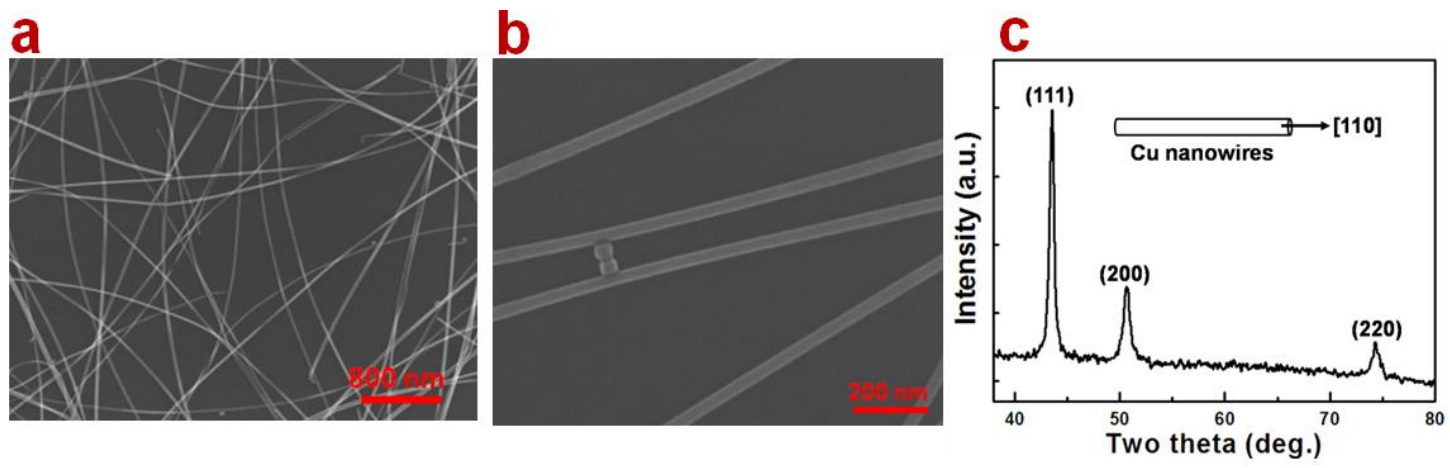

Fig. S2. SEM images (a) and (b), and XRD spectrum (c) of Cu NWs. The average diameter of $\mathrm{Cu}$ NWs measured from SEM images is $16 \pm 3 \mathrm{~nm}$. The elongated direction $\mathrm{Cu}$ NWs is along [110]. 


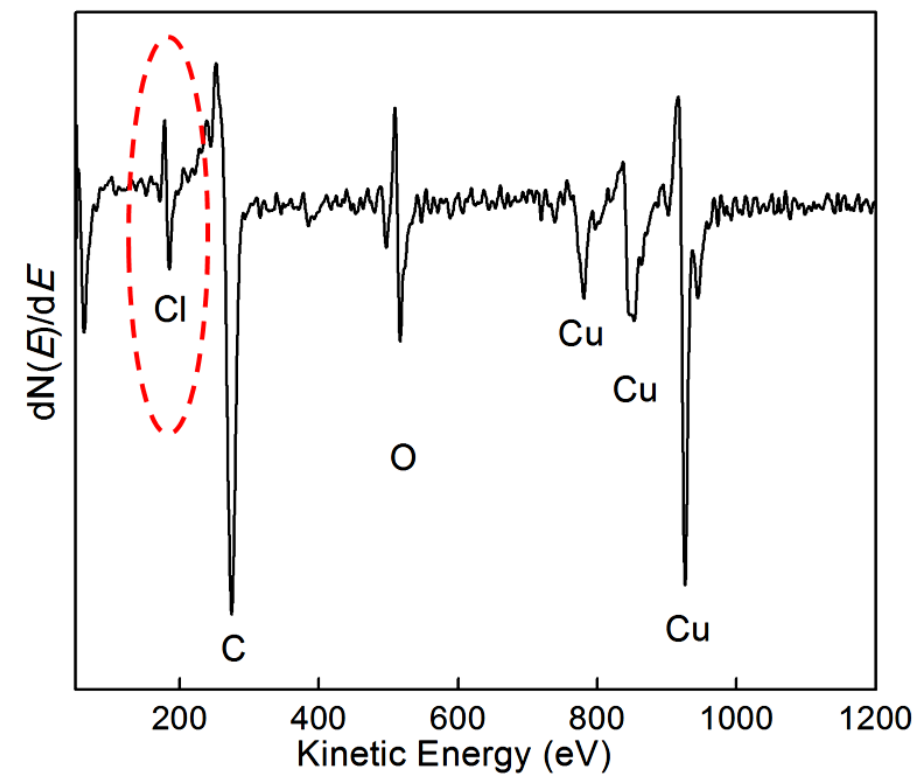

Fig. S3. Differential AES spectrum of Cu NSs. The the presence of $\mathrm{Cl}$ demonstrates the $\mathrm{Cl}^{-}$surfactants adsorbed on the $\mathrm{Cu}$ NS sidewall. The signal of oxygen may result from the surface-absorbed $\mathrm{CO}_{2}, \mathrm{H}_{2} \mathrm{O}$ and $\mathrm{O}_{2}$.

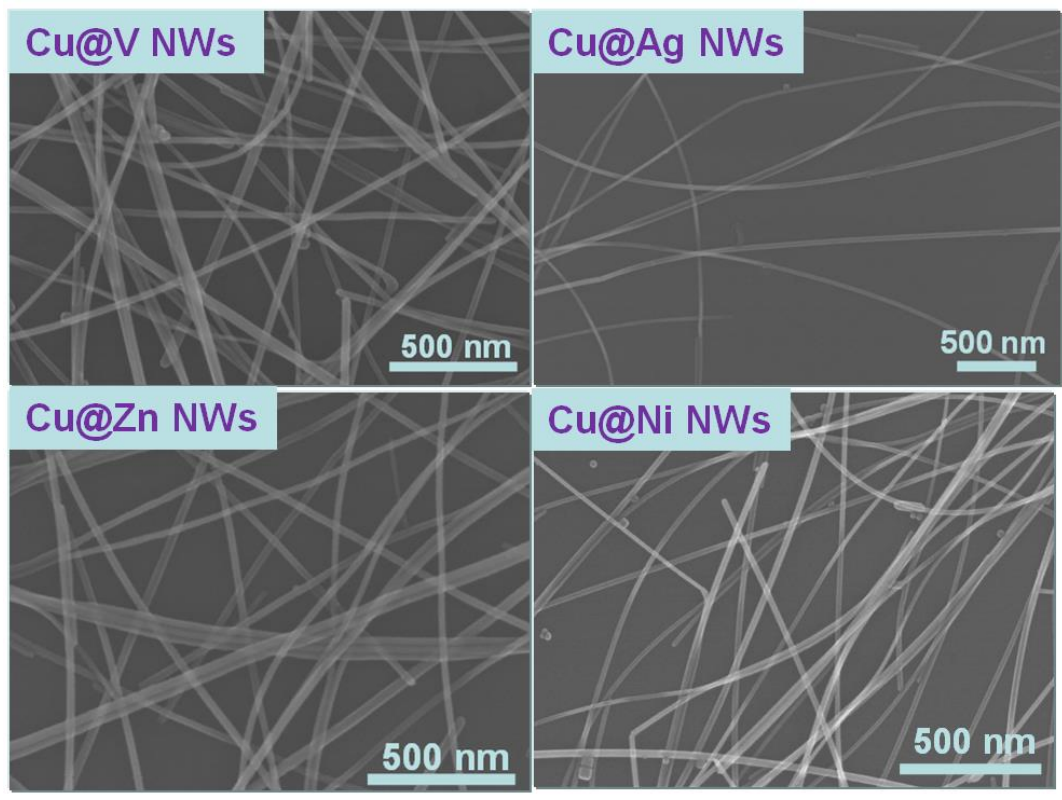

Fig.S4 SEM images of as-synthesized Cu@metal NWs. The stable metal coating based on the solution process could be seen from the uniform, smooth and particles-free morphology of the various core-shell NWs. 


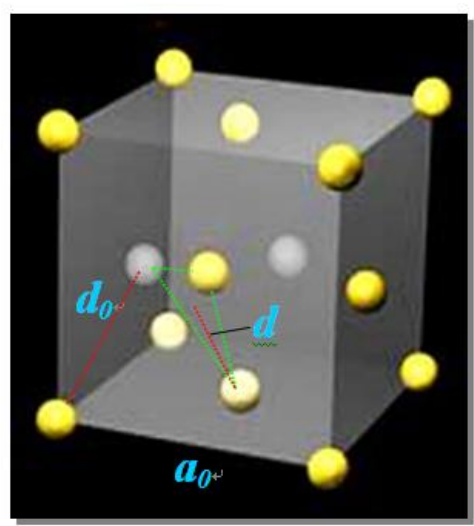

Fig. S5. Model of $\mathrm{Cu}$ facets in FCC structure. First-principles calculation of surface formation energy is performed based on this structure.

\section{First-Principles calculation of surface formation energies of various $\mathrm{Cu}$ facets}

The most stable bulk structure of copper is face-centered cubic (FCC), as shown in Fig. S5. Three major facets, (111), (110) and (100), were considered forming the clean surface on these orientations, by cleavage from FCC bulks. The model is constructed by repeated slab including 13 atomic metal layers and an about $18 \AA$ wide vacuum region. Calculations were performed within the framework of density functional theory, by using the Vienna ab initio simulation package (VASP). The projector augmented wave method was used and the plane-wave cutoff energy was set to 550 $\mathrm{eV}$. The exchangeand correlation potential was treated in a generalized gradient approximation (GGA-PBE) in the parameterization of Perdew, Burke, and Ernzerhof. Four to five topmost surface atomic layers were allowed for geometric optimization along the normal direction. The surface formation energies were obtained based on these relaxed clean surface models and with respect to the ideal bulk structure, whose equilibrium lattice constants were fully optimized as well. Important structural constants were obtained and compared with literatures, as listed in Table S1.

Table S1. Important constants of copper crystal. Lattice constants in $\AA$, and electronegativity $\alpha$ in Pauling scale. * Reported data in literatures.

\begin{tabular}{|c|c|c|c|c|c|}
\hline & $a_{0}$ & $a_{0}$ & $d_{0}$ & $d$ & $\alpha$ \\
\hline$\overline{\mathrm{Cu}}$ & 3.61 & 3.636 & 2.571 & 1.484 & 1.90 \\
\hline
\end{tabular}

Surface formation energy generally can be deduced by using the following equation,

$$
\sigma=\frac{1}{2 A}\left[E_{\text {slab }}-N \cdot E_{\text {bulk }}\right]
$$

where $A$ stands for the number of atoms on each surface, $E_{\text {slab }}$ is the total energy of the 
slab, $\mathrm{N}$ stands for the number of atoms in the slab, and $E_{b u l k}$ is the energy per atom in bulk. Usually, the smaller the surface energy is, the stabler the surface would be. The growth rate will also be higher. By using Eq. (1), the surface formation energies of different facets of $\mathrm{Cu}$ were calculated and summarized in Table S2.

Table S2. Surface formation energy of various facets of $\mathrm{Cu}$, in eV/atom.

\begin{tabular}{llll}
\hline \hline & $(111)$ & $(100)$ & $(110)$ \\
\hline $\mathrm{Cu}$ & 0.423 & 0.581 & 0.874 \\
\hline
\end{tabular}

a

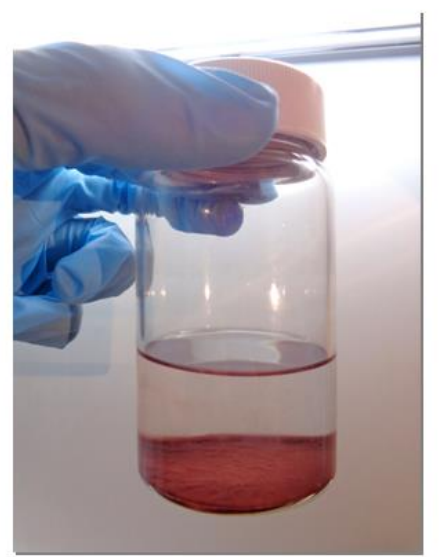

b

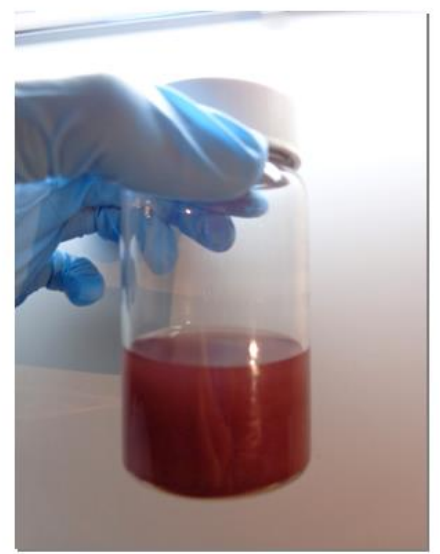

Fig. S6. Photographs of Cu NWs kept in hexane solution for more than two months (a) and after bath sonication (b), which shows excellent dispersion and stability.
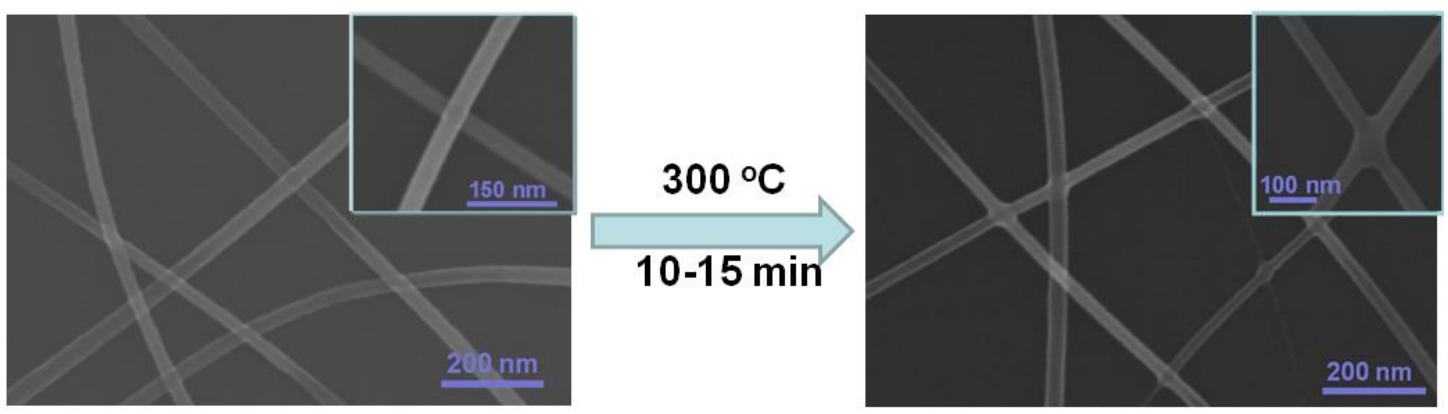

Fig. S7. Thermal annealing process of $\mathrm{Cu} @$ metal NWs for conductive film: SEM images before (left) and after (right) annealing. The junctions are welded at $300{ }^{\circ} \mathrm{C}$ for 10-15 minutes. The nanowelding process enhances the electronic conductivity by reducing the contact resistance. 


\section{$100 \mathrm{~nm}$}

Fig. S8. AFM scanning of the welded junctions between $\mathrm{Cu} @ \mathrm{Ti}$ NWs network. The firm fuse of the touching junction could be clearly seen.
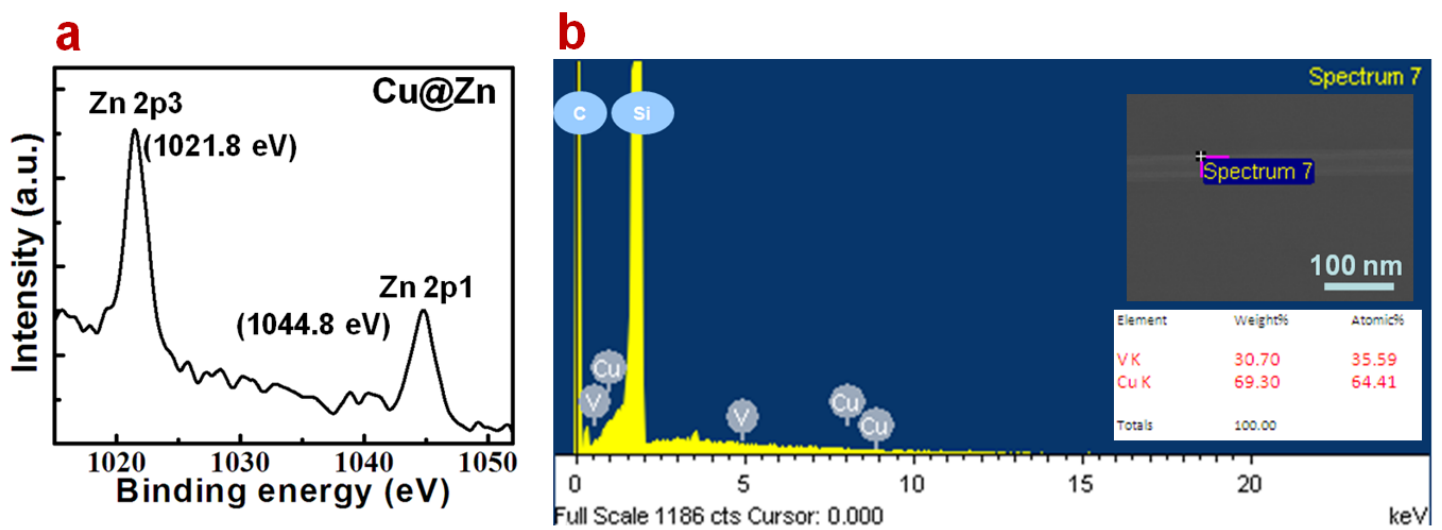

Fig. S9. XPS spectroscopy (a) of $\mathrm{Zn}_{2 \mathrm{p}}$ for $\mathrm{Cu} @ \mathrm{Zn} \mathrm{NWs}$ and EDS (b) for $\mathrm{Cu} @ \mathrm{~V}$ NWs on silicon substrate. The coating of $\mathrm{Zn}$ and $\mathrm{V}$ on $\mathrm{Cu}$ NWs and formation of core-shell structure is further confirmed.

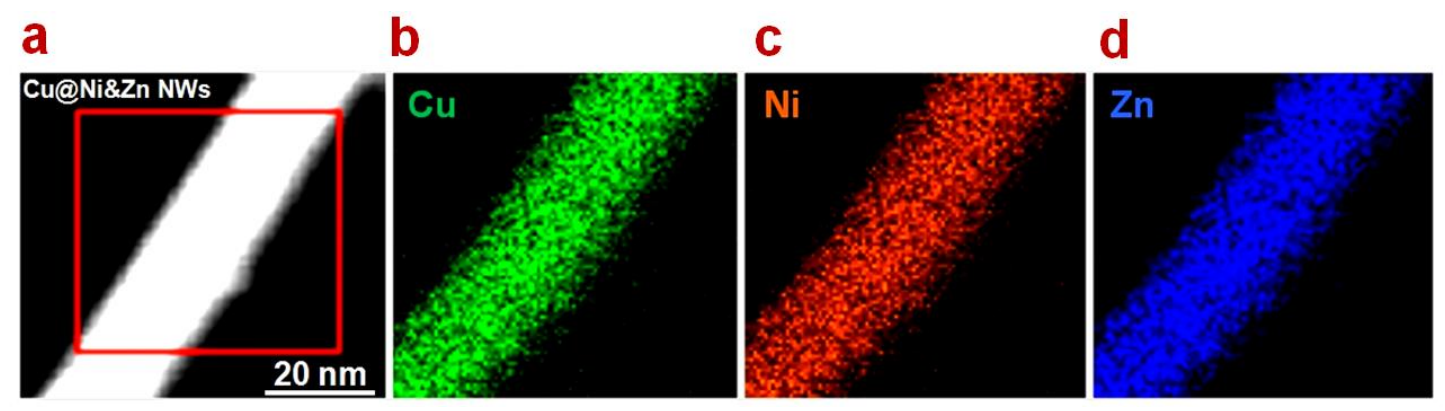

Fig. S10. STEM image of Cu@NiZn NW (a) and EDS elemental mapping iamges (b-d) for $\mathrm{Cu}, \mathrm{Ni}$ and $\mathrm{Zn}$, respectively. The uniform coating of $\mathrm{Ni}$ and $\mathrm{Zn}$ on the $\mathrm{Cu}$ NW surface and formation of alloy in the shell layer is confirmed. 


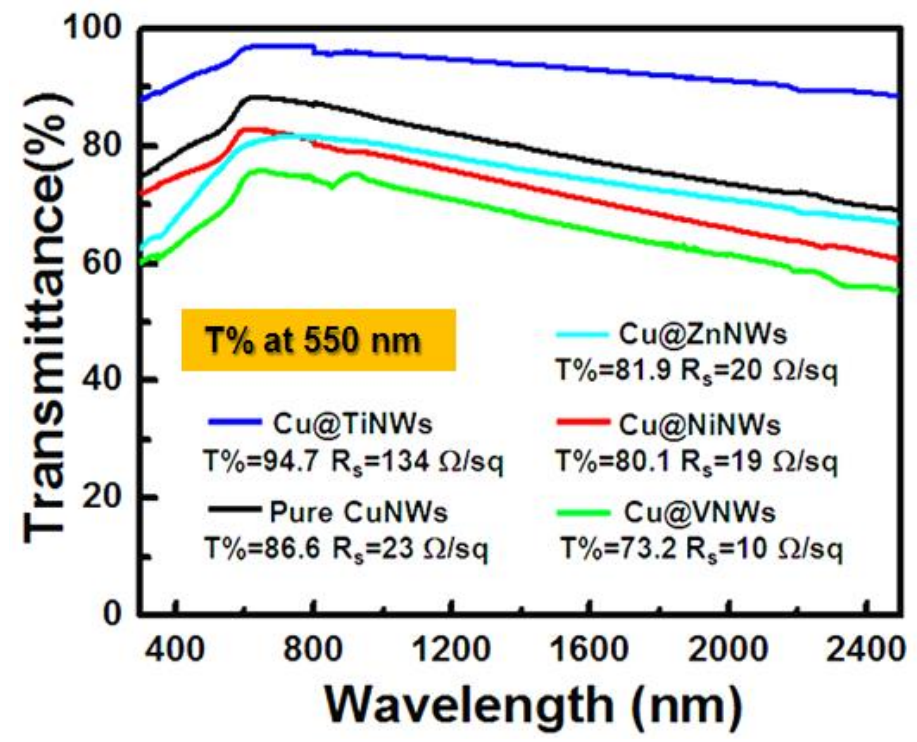

Fig. S11. Transmittance versus wavelength for pure $\mathrm{Cu}$ NWs and $\mathrm{Cu} @$ metal NWs. The high transmittance in a wide wavelength range for UV to IR demonstrates the excellent optical transparency property for NWs as TCEs.

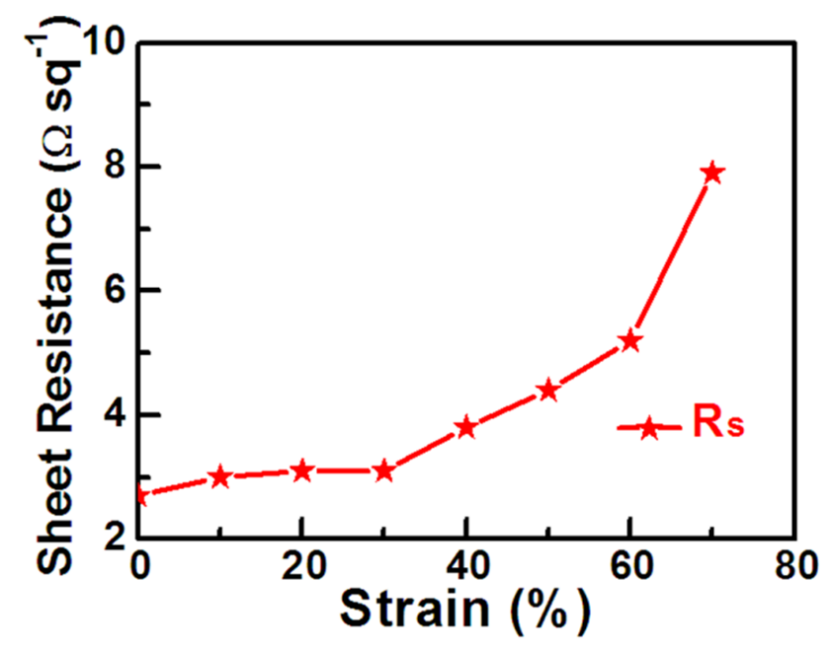

Fig. S12. Sheet resistance as a function of stretching strain after each strain release for the sample Cu@Ti NWs/silica gel plate. These resistances were measured for the first stretching cycle. After the second cycle, resistance becomes recoverable. 

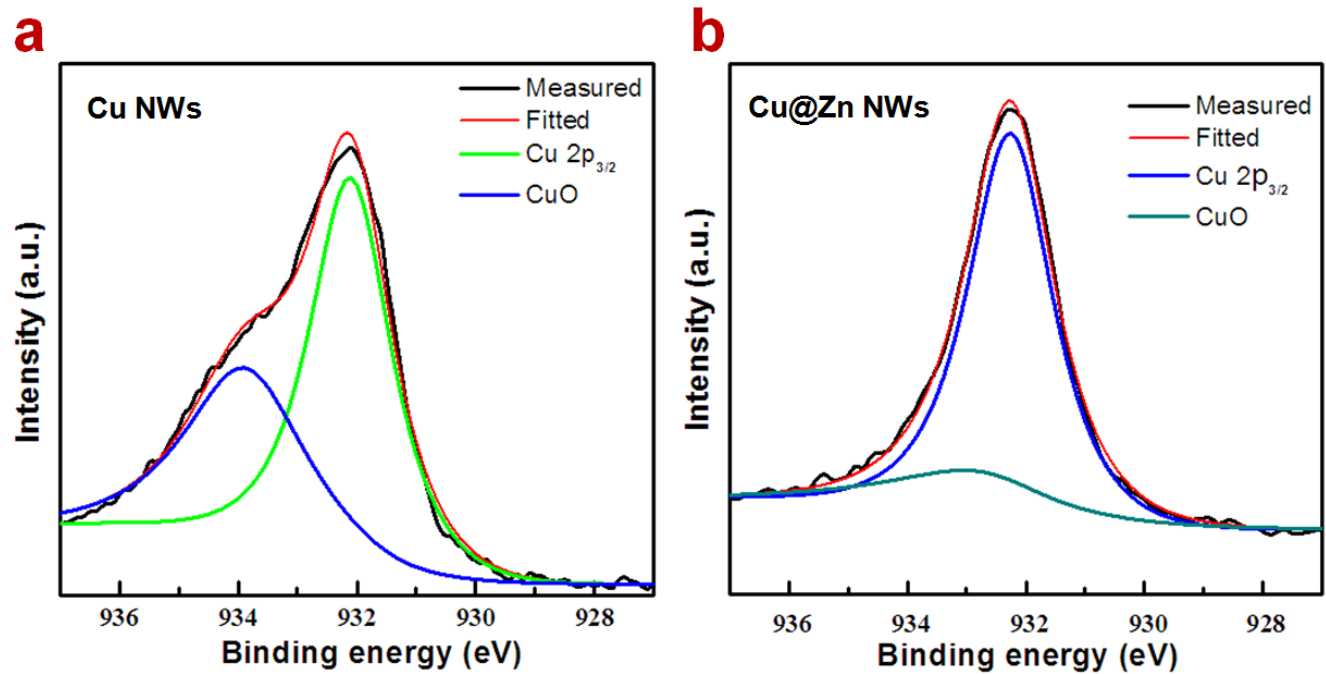

Fig. S13 XPS spectra of Cu NWs (a) and Cu@Zn (b) NWs films after stability tests.
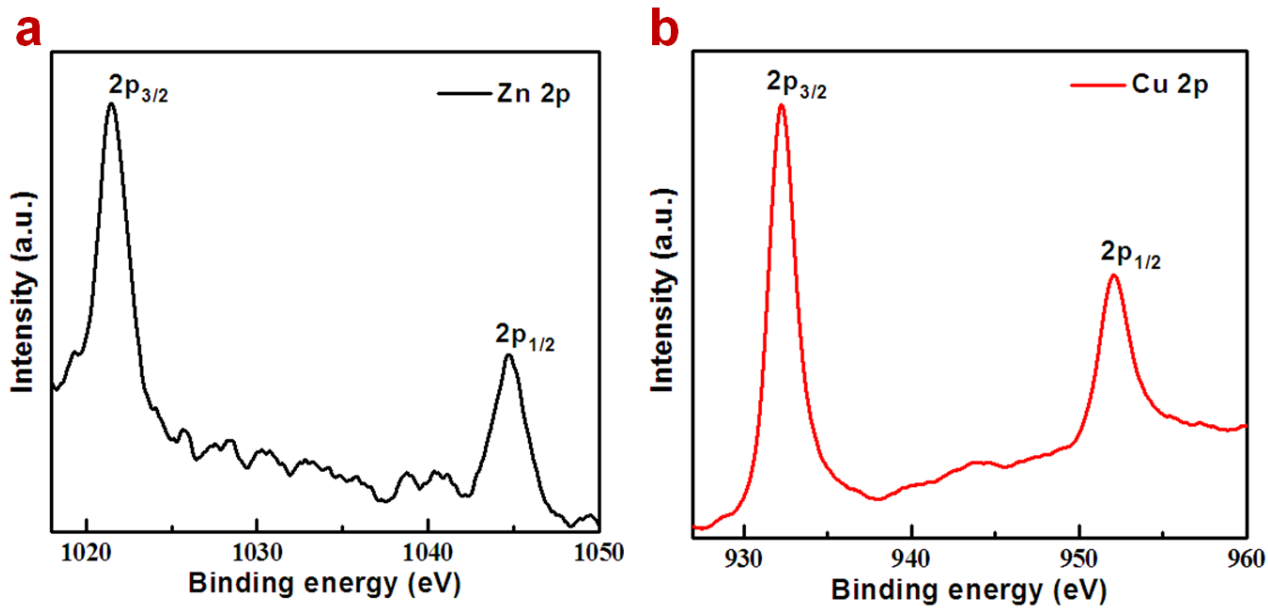

Fig. S14 XPS spectra of Zn2p (a) and Cu2p (b) for Cu@Zn NWs, which confirms the formation of $\mathrm{Zn}$ shell layer. 


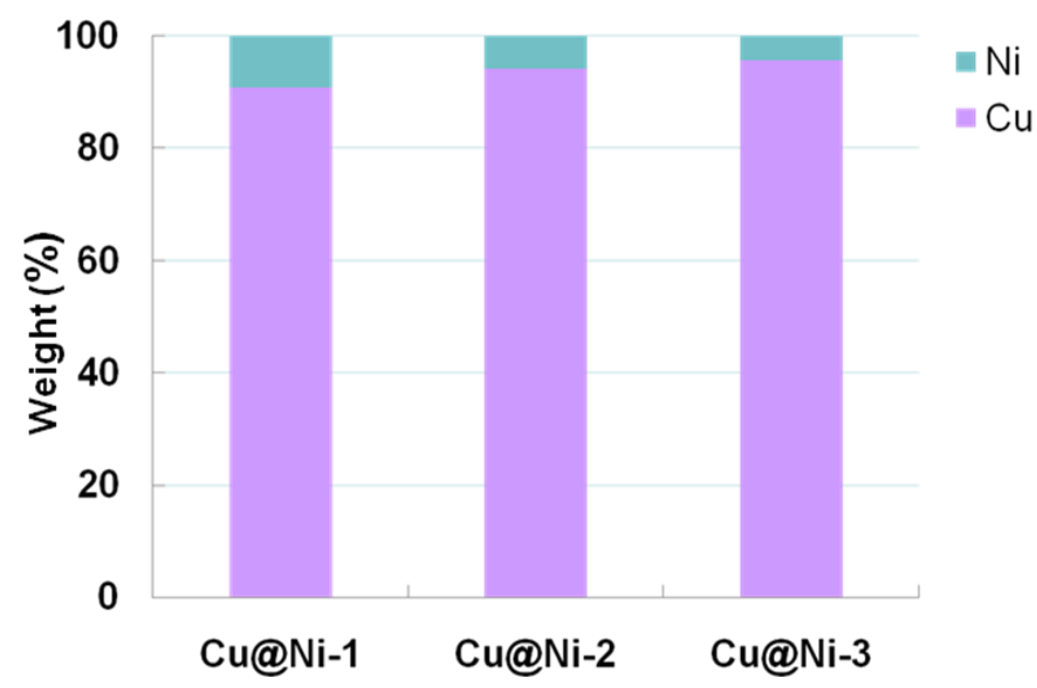

Fig. S15 Weight percentage of $\mathrm{Cu}$ and $\mathrm{Ni}$ in $\mathrm{Cu} @ \mathrm{Ni}$ NWs with different $\mathrm{Ni} / \mathrm{Cu}$ molar ratio $(\mathrm{Cu} @ \mathrm{Ni}-1,2,3)$.

\section{Supporting Movie caption:}

Movie S1. The operation of transparent dimmer with $\mathrm{Cu} @ \mathrm{Ti}$ NWs for red LED lighting. The initial working voltage is set to $1.8 \mathrm{~V}$ (DC). The dimmer is stretched by tensile strain from $0 \%$ gradually up to $60 \%$. The luminance of LED can be tuned by controlling the working current. After release of the stretching strain, the LED brightness is well recovered to the initial state. 